\title{
Commentary: NO matter what you call it, "conditioning" the spinal cord preventing paraplegia is worthwhile
}

\author{
Muhammad Aftab, MD, and T. Brett Reece, MD
}

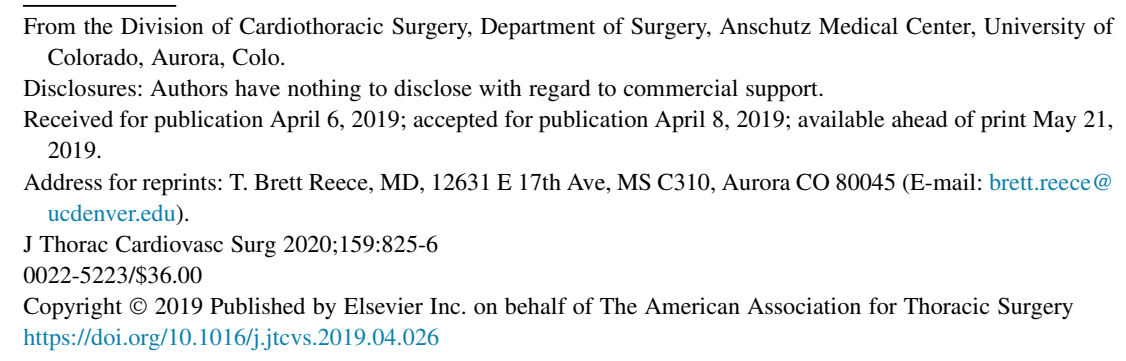

Postoperative spinal cord ischemia, resulting in paraplegia, remains one of the most dreaded complications of thoracic aortic surgery. Paraplegia results in increased mortality and poor long-term survival. Despite recent advancements in the perioperative strategies for spinal cord protection, composite and permanent spinal cord deficits occurred in $9.6 \%$ and $5.4 \%$ of 3309 thoracoabdominal aortic aneurysm repairs in the best hands. The delayed spinal cord deficit resulting in permanent paraplegia and paraparesis occurred in $1.4 \%$ and $1.6 \%$ of these patients. ${ }^{1}$ These complications warrant exploration into novel methods of spinal cord protection, beyond perioperative perfusion strategies, cerebrospinal fluid drainage, and utility of collateralization. In their publication in this issue of the Journal, Li and coworkers ${ }^{2}$ demonstrated that in a rabbit model, spinal cord postconditioning by stimulation to $2 \mathrm{~Hz}$ offers neuroprotective effects against ischemia-reperfusion injury. This same group of authors previously reported that spinal cord stimulation (SCS) postconditioning at both $2 \mathrm{~Hz}$ and $50 \mathrm{~Hz}$ offers neuroprotective effects; however, these effects were more pronounced in the $2-\mathrm{Hz}$ group.

$\mathrm{Li}$ and coworkers ${ }^{2}$ sought to evaluate the outcomes in terms of hind limb neurologic functions, the number of $\alpha$ motor neurons, terminal deoxynucleotidyl transferase deoxyuridine-5'-triphosphate nick end labeling-positive neuron rates and ratios of the microglia area to the anterior horn area (microglia area ratios). The rabbits of the $2-\mathrm{Hz}$ group fared significantly better than the control group in all end points. The rabbits treated with $\alpha 7$ nicotinic acetylcholine receptor $(\alpha 7 \mathrm{nAchR})$ antagonist ( $\alpha$ - bungarotoxin group) fared significantly worse than the control group. The outcomes of combined treatment with $2-\mathrm{Hz}$ stimulation and $\alpha$ - bungarotoxin were not significantly different from those of the control group, except for significantly lower neuronal apoptosis, as demonstrated by terminal deoxynucleotidyl transferase deoxyuridine-5'-triphosphate nick end labeling-positive neuron rates. $\mathrm{Li}$ and coworkers ${ }^{2}$ concluded that this protection is partially mediated by

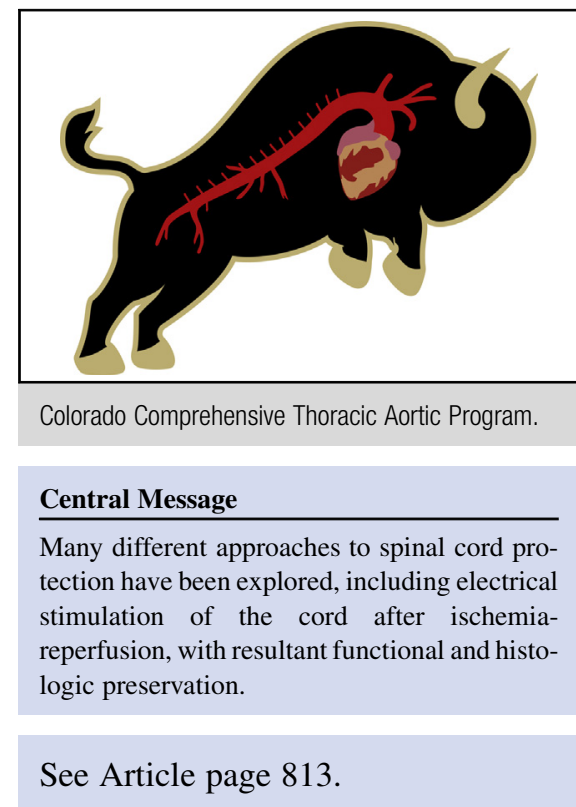

activation of $\alpha 7 \mathrm{nAchR}$. The approach is outside the box of our usual thinking, but it produces promising functional protection.

Spinal cord protection in the perioperative period of complex aortic intervention is achieved by a complex interplay of ischemic and inflammatory factors. The exact mechanism of protection remains unclear with respect postischemic stimulation, whether through direct protection of the neuron from ischemia-reperfusion injury or limiting apoptosis through an unknown means. If this is the case, could early mobilization and spinal cord stimulation of patients after aortic surgery offer a similar advantage? Others have shown the effects of activation of $\alpha 7 \mathrm{nAChRs}$ on cerebral perivascular sympathetic nerves. It enhances the calcium influx and promotes the release of norepinephrine, resulting in nitric oxide release and vasodilation. ${ }^{4}$ It is unknown whether SCS induces local vasodilation similar to that of the cerebral vasculature and thus enhances the collateralization of spinal cord capillary network by release of local hormones. The entire mechanism of this neuroprotection offered by SCS postconditioning remains to be elucidated. Furthermore, it would be interesting to see whether agonists of $\alpha 7 \mathrm{nAchRs}$, such as nicotine, GTS-21, and PNU-282987, can offer similar neuroprotection from ischemia-reperfusion injury. ${ }^{5,6}$

SCS is used selectively in clinical practice to manage chronic pain in some patients as a last resort. From a 
translational standpoint, the implantation of epidural stimulation electrode appears a quite complex procedure. To make this a viable option, researchers should explore less invasive and percutaneous alternatives that can offer equivalent neuroprotection to SCS postconditioning.

To improve neurologic outcomes after extensive thoracoabdominal repairs and offer the best protection from the spinal cord ischemic injury, there is a need to explore novel mechanisms and molecular targets, focusing on combining both presurgical ischemic tolerance and postconditioning. There is a certainly a need to develop innovative techniques that can induce spinal cord precollateralization and thus offer superior protection from ischemic-reperfusion injury beyond the current perioperative strategies.

\section{References}

1. Coselli JS, LeMaire SA, Preventza O, de la Cruz KI, Cooley DA, Price MD, et al. Outcomes of 3309 thoracoabdominal aortic aneurysm repairs. J Thorac Cardiovasc Surg. 2016;151:1323-37.

2. Li H, Dong X, Cheng W, Jin M, Zheng D. Neuroprotective mechanism involved in spinal cord stimulation postconditioning. J Thorac Cardiovasc Surg. 2020; 159:813-24.e1.

3. Li H, Dong X, Jin M, Cheng W. The protective effect of spinal cord stimulation postconditioning against spinal cord ischemia/reperfusion injury in rabbits. $\mathrm{Neu}$ romodulation. 2018:21:448-56.

4. Si ML, Lee TJ. $\alpha_{7}$-nicotinic acetylcholine receptors on cerebral perivascular sympathetic nerves mediate choline-induced nitrergic neurogenic vasodilation. Circ Res. 2002;91:62-9.

5. Yamanaka K, Eldeiry M, Aftab M, Ryan TJ, Meng X, Weyant MJ, et al. Synergetic induction of NGF with diazoxide and erythropoietin attenuates spinal cord ischemic injury. J Surg Res. 2019;233:124-31.

6. Narla S, Klejbor I, Birkaya B, Lee YW, Morys J, Stachowiak EK, et al. $\alpha 7$ Nicotinic receptor agonist reactivates neurogenesis in adult brain. Biochem Pharmacol. 2013;86:1099-104. 